\title{
Article \\ Bearing Fault Reconstruction Diagnosis Method Based on ResNet-152 with Multi-Scale Stacked Receptive Field
}

\author{
Hu Yu, Xiaodong Miao *D and Hua Wang \\ School of Mechanical and Power Engineering, Nanjing Tech University, Nanjing 211816, China; \\ 202061207070@njtech.edu.cn (H.Y.); wanghua@njtech.edu.cn (H.W.) \\ * Correspondence: mxiaodong@njtech.edu.cn
}

check for

updates

Citation: Yu, H.; Miao, X.; Wang, H. Bearing Fault Reconstruction Diagnosis Method Based on ResNet-152 with Multi-Scale Stacked Receptive Field. Sensors 2022, 22, 1705. https://doi.org/10.3390/ s22051705

Academic Editors: Lang $\mathrm{Xu}$ and Steven Chatterton

Received: 30 January 2022

Accepted: 17 February 2022

Published: 22 February 2022

Publisher's Note: MDPI stays neutral with regard to jurisdictional claims in published maps and institutional affiliations.

Copyright: (C) 2022 by the authors. Licensee MDPI, Basel, Switzerland. This article is an open access article distributed under the terms and conditions of the Creative Commons Attribution (CC BY) license (https:// creativecommons.org/licenses/by/ $4.0 /)$.

\begin{abstract}
The axle box in the bogie system of subway trains is a key component connecting primary damper and the axle. In order to extract deep features and large-scale fault features for rapid diagnosis, a novel fault reconstruction characteristics classification method based on deep residual network with a multi-scale stacked receptive field for rolling bearings of a subway train axle box is proposed. Firstly, multi-layer stacked convolutional kernels and methods to insert them into ultradeep residual networks are developed. Then, the original vibration signals of four fault characteristics acquired are reconstructed with a Gramian angular summation field and trainable large-scale 2D time-series images are obtained. In the end, the experimental results show that ResNet-152-MSRF has a low complexity of network structure, less trainable parameters than general convolutional neural networks, and no significant increase in network parameters and calculation time after embedding multi-layer stacked convolutional kernels. Moreover, there is a significant improvement in accuracy compared to lower depths, and a slight improvement in accuracy compared to networks than unembedded multi-layer stacked convolutional kernels.
\end{abstract}

Keywords: subway train; fault diagnosis; data reconstruction; deep learning; residual neural network; Gramian angular field

\section{Introduction}

Subway trains are integral to traffic systems, modernization, and urban culture [1-3]. However, because these axle boxes of a subway train support the whole weight of the subway vehicle and ensure the reliability of a subway train [4,5], and the rolling bearings are the vitally important component to transfer loads and torque through which are filtered by an air spring to shaft. Hence, failures unavoidably occur in rolling bearings and result in economic loss or even human casualties. As a result, fast and accurate fault diagnosis of axle box bearings can be used to maintain the smooth operation of urban rail transit and extend service time as well as ensure travel safety.

The fault diagnosis methods used mainly for rolling bearings can be classified into two categories, vibration-based signal analysis and machine-learning-powered methods [6-10]. In general, vibration-based signal analysis methods detect faults by extracting fault-related vibration components and characteristic frequency. However, vehicle-mounted sensors used to extract other irrelevant vibration signals including the shaft and gearbox, etc., when subway trains operate at high speeds. Hence, in the early stage of faults, bearing-related fault signals used to be overwhelmed by overstated other components and harmonics or environment noise. Therefore, it is hard to extract pure fault-related vibration signal by traditional vibration-based signal analysis.

Machine-leaning-powered fault diagnosis methods detect faults by extracting a series of statistical parameters (e.g., such as kurtosis, root mean square, energy and entropy.) to represent bearings' health states. Meanwhile, these parameters can be used to train classifiers (e.g., such as a support vector machine (SVM), a deep neural network (DNN), 
or a Bayes network) to classify different fault characteristics. Among them, SVM is a class of generalized linear classifiers that perform binary classification of data in a supervised learning manner, neural networks are based on extensions of perceptrons, while DNN can be understood as neural networks with many hidden layers. Nevertheless, the extracted statistical parameters cannot ensure the accuracy of distinguishing different faults. Therefore, finding suitable training parameters to train traditional classifiers is a long-term challenge for machine-learning-powered fault diagnosis methods [11].

In recent years, deep learning (DL) methods, which take vibration-related signals as input data, has been applied in various fields [12-16]. For example, S. Roy et al. [12] applied the successful application of DL in medical imaging to COVID-19 as well as paved the way to future research on DL for the assisted diagnosis of COVID-19 from medicine imaging datasets. K.B. Lee et al. and H. S. DIKBAYIR et al. [13-15] detected vehicles in different complex driving environments based on DL. For fault-related signal features extraction, traditional machine-learning-powered methods, which rely on fault-related preprocessing, are the lack of multiple levels of nonlinear transformations [17]. DL cannot only adaptively extract deep features of fault characteristics from anc input layer but can also ease the difficulty of parameters optimizations.

Among the DL theoretical methods, diagnostic models with classical convolutional neural network (CNN) structure are the most widely used, such as Alex Net, VGG, etc. However, because of the big data with large volume, vary modalities, fast generation and large value but low density, these network models have no choice but to improve the depth to parse the massive data, so it will cause huge training parameters and overfitting. By contrast, deep residual networks (ResNets) are an effective variant of CNNs, which can use identity shortcuts to ease the difficulty of parameters optimization [18-21]. ResNets and their variants have applied for fault diagnosis in a few papers [22-28]. For instance, C. Zhou et al. [24] analyzed the COVID-19 chest X-raay images based on image regrouping and ResNet-SVM. As a consequence, this method can reach 93\% accuracy on a relatively small dataset. M. Zhao et al. [27] proposed deep residual shrinkage network (DRSN), which is an evolution of ResNets. Compared with structure of ResNets, DRSN has a shrinkage block (soft threshold function) and show that the method is effective for high noise fault diagnosis.

The developed residual neural networks can adapt to large-scale data and have good nonlinear expression capability, but a large number of research is based on 1D fault vibration signals, which makes full use of the self-extraction capability of DL but also limits the diagnostic accuracy. This article develops a fault reconstruction characteristics classification method using ResNet-152 inserted with multi-layers convolutional kernels. The data is structured by convolutional units with multi-layer stacked convolutional kernels to enhance the nonlinear representation. One-dimensional vibration signals processed by Gramian angular summation field (GASF) are easier to manipulate by convolutional layers. The main contributions of this paper are as follows:

(1) Three-layers stacked convolutional kernels are inserted into ultra-deep ResNets to replace large-size or less-layers convolutional kernels to improve the nonlinear representation of feature images;

(2) The fault datasets are reconstructed to increase the data scale and retain the temporal features in the fault data, while reducing the difficulty of the convolution process;

(3) Research on axle box bearings for subway trains to improve the efficiency and accuracy of diagnosis of this component.

Additionally, this study verifies the role of superimposed convolution kernels in specific objects for the first time on the basis of the theory; another novelty is the training of deep learning networks using reconstructed fault feature signals, as researchers have overlooked the importance of modest feature engineering while focusing too much on the powerful learning ability of deep learning.

This paper is organized as follows: in Section 2, ResNets-related methods are introduced. In Section 3, details of the design of fundamental architectures for ResNet-152-MSRF, 
data reconstruction methods, experimental protocols and complete experimental results are presented. In Section 4, the experimental results are summarized, and the advantages and disadvantages of each model are sorted out.

\section{Basic Components}

\subsection{Basic Structure of Residual Neural Networks}

ResNets share many of the same components as traditional CNNs, such as convolution layers, rectifier linear unit (ReLU), activation function, batch normalization (BN), loss function and pooling layers et al. In the fact, the pooling layer, which downsamples fault-related features to submit to the next block, can be used or not in many deep neural networks. The theories of these basic components are described as follows.

The convolution operation in a CNN is the key component of the entire network and is the essential difference from a fully connected (FC) neural network. The convolutional layer in a CNN can effectively reduce the amount of the trainable parameters, so that the training speed of the model is greatly improved. In a neural network, the fewer the trainable parameters, the less likely the network will be over-fitted. The formula for the convolution operation can be expressed as follows:

$$
z_{u, v}^{(l)}=\sum_{i=-\infty}^{\infty} \sum_{j=-\infty}^{\infty} x_{i+u, j+v}^{(l-1)} \cdot k_{r o t}(l) \cdot X(i, j)+b^{(l)}
$$

where $x_{i+u, j+v}^{(l-1)}$ is the $(l-1)$ th channel of the input feature map, $k_{r o t i, j}^{(l)}$ is the $l$ th corresponding convolution kernel, $X(i, j)$ is the activation function of the corresponding layer, $b^{(l)}$ is the corresponding bias term, $i, j$ are the relative positions during feature mapping, $z_{u, v}^{(l)}$ is the $l$ th channel of output feature map. The convolution operation can be repeated several times to obtain a large number of feature maps.

$\mathrm{BN}$ is an important technique for normalizing feature data as a trainable process to be inserted into the deep learning architecture [29,30]. Deep networks training is a complex process, whenever a small change occurs in the first few layers of the network, then the later layers will be cumulatively amplified down. Hence, the purpose of BN is to reduce the internal covariate shift, in which updates of the front layer training parameters will lead to changes in the distribution of the back layer input data. As a matter of fact, BN force the distribution of the input value of any neuron in each layer of the neural network back to a standard normal distribution with a mean of zero and a variance of one, so that the activation input value falls in the region where the nonlinear function is more sensitive to the input. BN operation is expressed as follows:

$$
\begin{aligned}
\mu_{\beta} & =\frac{1}{m} \sum_{i=1}^{m} x_{i} \\
\sigma_{\beta}^{2} & =\frac{1}{m} \sum_{i=1}^{m}\left(x_{i}-\mu_{\beta}\right)^{2} \\
\hat{x}_{i} & =\frac{x_{i}-\mu_{\beta}}{\sqrt{\sigma_{\beta}^{2}+\varepsilon}} \\
y_{i} & =\gamma \hat{x}_{i}+\beta \rightarrow B N_{\gamma, \beta}\left(x_{i}\right)
\end{aligned}
$$

where $x_{i}$ and $y_{i}$ represent the input and output feature of the $i$ th observation in a mini-batch. $\gamma$ and $\beta$ are two trainable parameters to adjust the distribution. $\varepsilon$ is a constant that tends to zero.

Loss function is used to measure the quality of a set of parameters by comparing the difference between the expected output and the true output. In multi-category tasks, the cross-entropy error used to be the objected function to be minimized. Compared with other traditional error functions, cross-entropy can promise a higher training efficiency. Apart from that, in order to strengthen the feature, cross-entropy is usually used with the softmax 
function to map the output from zero to one. Then softmax function can be expressed as follows:

$$
\begin{aligned}
& p(y \mid x)=\frac{e^{\left(W_{y} \cdot x\right)}}{\sum_{c=1}^{C} e^{W_{c} \cdot x}} \leftarrow W_{y} \cdot x=\sum_{i=1}^{d} W_{y i} \cdot x_{i}=f_{y} \\
& \Downarrow(y \mid x)=\frac{e^{\left(f_{y}\right)}}{\sum_{c=1}^{C} e^{\left(f_{c}\right)}} \rightarrow \operatorname{softmax}(f)_{y}
\end{aligned}
$$

the first step is to take the $y$ th row of $W$ and multiply that row with $x$ as well as compute for all $f_{c}$ for $c=1, \ldots, C$, and then apply the softmax function to get a normalized probability. Cross-entropy is expressed as follows:

$$
H(p, q)=-\sum_{i=1}^{n} p\left(x_{i}\right) \log q\left(x_{i}\right)
$$

where $p\left(x_{i}\right)$ is the $i$ th actual probability of observation. After calculating the cross-entropy error, the gradient descent algorithm is used to optimize the parameters, and then the network is fully trained after several iterations.

\subsection{Insertion of Multi-Scale Superimposed Receptive Field}

In this section, the motivation for fault characteristics reconstruction and multi-scale superimposed receptive field that insert into the architecture of deep residual network are introduced.

Receptive field is the convolutional kernel which realize the local perception of the corresponding input, the implementation is a weighted summation over a local region of the input. The size of the convolution kernel must be larger than 1 to have the effect of enhancing the perceptual field, so that the most commonly used convolutional kernel for feature extraction cannot be 1 . Convolution kernels of even size cannot guarantee that the input feature map size and output feature map size remain unchanged even if padding is added symmetrically (e.g., if the input is $4 \times 4$ and the convolution kernel size is $2 \times 2$ and padding is 1 on each side, there will be a total of 5 outputs after sliding, which will not correspond to the input). Compared with bigger convolution kernels, multi-layer stacked small-sized convolution kernels have more activation functions, richer features and greater discernment. Convolution operation is accompanied by an activation function, and the use of more convolution kernels can make the decision function more discriminative.

Multi-layer stacked convolutional kernel replacement for large size convolution kernel involves parameter calculation. Table 1 shows the comparison of whether stacked convolution is used or not for different kinds of networks or for the same kind of networks with different depths, including VGG-16, VGG-19, ResNet-50, ResNet-152. As shown in Table 1, multi-layer stacked convolutional kernels have a larger number of parameters compared to large size convolutional kernels and few-layer convolutional kernels, but parameters growth rates are all stable at less than $1 \%$, the replacement of $7 \times 7$ convolutional kernels with $3 \times 3+3 \times 3+3 \times 3$ stacked convolutional kernels in the VGG-19 network has the smallest parameter growth rate of $0.06 \%$, and the replacement of $5 \times 5$ convolutional kernels with $3 \times 3+3 \times 3$ stacked convolutional kernels in the ResNet-152 network has the largest parameter growth rate of $0.9 \%$.

Table 1. Comparison of the number of computational parameters by multi-layer stacked convolutional kernels inserted into different networks.

\begin{tabular}{ccccc}
\hline & $\mathbf{3} \times \mathbf{3 + 3} \times \mathbf{3}$ & $\mathbf{5} \times \mathbf{5}$ & $\mathbf{3} \times \mathbf{3 + 3} \times \mathbf{3 + 3} \times \mathbf{3}$ & $\mathbf{7} \times \mathbf{7}$ \\
\hline VGG-16 & $18,952,131$ & $18,310,787$ & $19,137,027$ & $19,102,595$ \\
VGG-19 & $53,595,203$ & $53,544,835$ & $53,779,715$ & $53,746,051$ \\
ResNet-50 & 762,691 & 714,703 & $1,234,093$ & $1,197,084$ \\
ResNet-152 & $2,419,171$ & $2,397,061$ & $6,679,779$ & $6,434,577$ \\
\hline
\end{tabular}


The superiority of the residual network can also be seen in Table 1, where the number of trainable parameters of the ResNet with a depth of 152 layers is less than $20 \%$ of that of the VGG network with only 16 or 19 layers, so that ResNets are well suited for embedding stacked convolutional kernels, which stabilize the number of parameters while keeping the network lightweight.

\section{Design of Fundamental Architectures for ResNet-152-MSRF}

In this section, the architecture of ResNet-152-MSRF are elaborated.

The core part of the developed ResNet-152-MSRF is shown in Figure 1, the convolution process is achieved by stacked convolutional kernels. The input image is output to the next stage with tensor data with deep nonlinear features by the action of a three-layer stacked convolution kernel. Neural networks gradually lose local features at each layer through pooling as the depth increases, which is fatal for fault diagnosis. ResNets, on the other hand, lead to ultra-deep network structures. ResNet-152-MSRF has 152 convolutional layers, and each convolutional layer performs 3 nonlinear transformations because of the embedded 3-layer stacked convolutional kernel, and then average pooling is used between each layer as well as the number of feature channels increases and the feature size decreases. In Figure 2 is the overall architecture of ResNet-152-MSRF. The input features of the previous convolutional layer are added to the output features by identity shortcutting, prerequisite is to ensure the same shape (e.g., the input shape of the previous layer is $64 \times 64 \times 16$, then the output feature shape is the same). Dropout(0.5) function is used between each convolutional layer to randomly reduce the number of neurons to prevent overfitting, 0.5 represents the random neuron discard rate. Finally, the fully connected layer is connected, and the number of output nodes is the same as the number of categories.

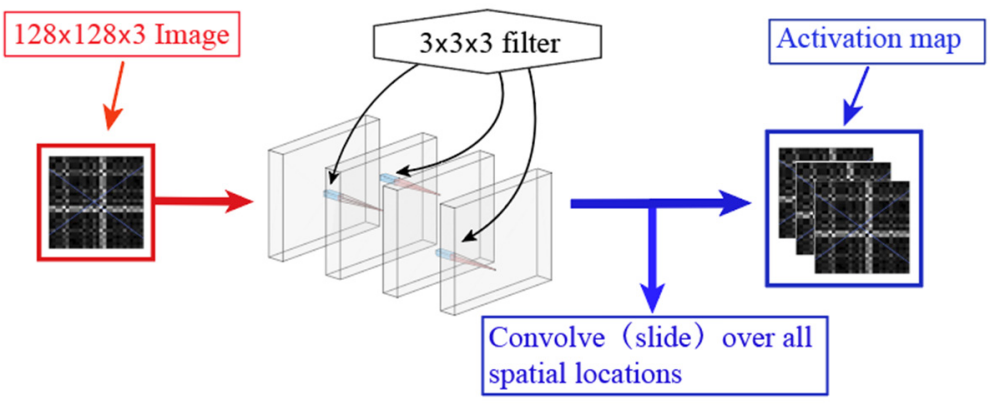

Figure 1. Convolutional process of multi-layer convolutional kernel embedded in residual network.

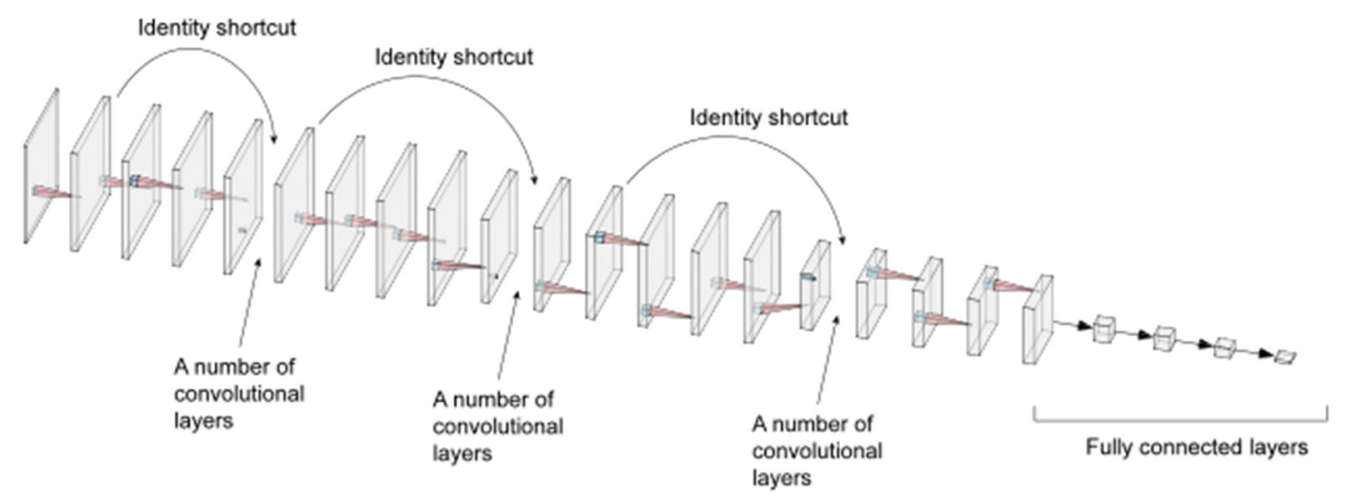

Figure 2. Overall architecture of ResNet-152-MSRF.

The advantage of using this architecture is that it can train large datasets well, and the network is deep enough to make sufficient nonlinear transformations to allow the computer to discriminate features. 


\section{Experiment Results}

\subsection{Data Collection and Processing}

The drive-train dynamic simulator used for this experimental data acquisition is shown in Figure 3. The entire device consists of a motor, an acceleration sensor, a magnetic brake and a subway train axle box. The load and speed conditions of the bearings in the axle box are determined by the motor, and the sampling frequency is determined by the acceleration sensor. The acceleration sensor is installed between the motor and the axle box (i.e., the input of torque) of the axle box, raw vibration data were collected for 4 health conditions with a sampling frequency of 12,000 Hz. As shown in Table 2, the four health conditions are summarized, including one healthy condition and three faults. The bearings under each health condition are subjected to different loads of $36 \mathrm{KN}$ and $72 \mathrm{KN}$, respectively.

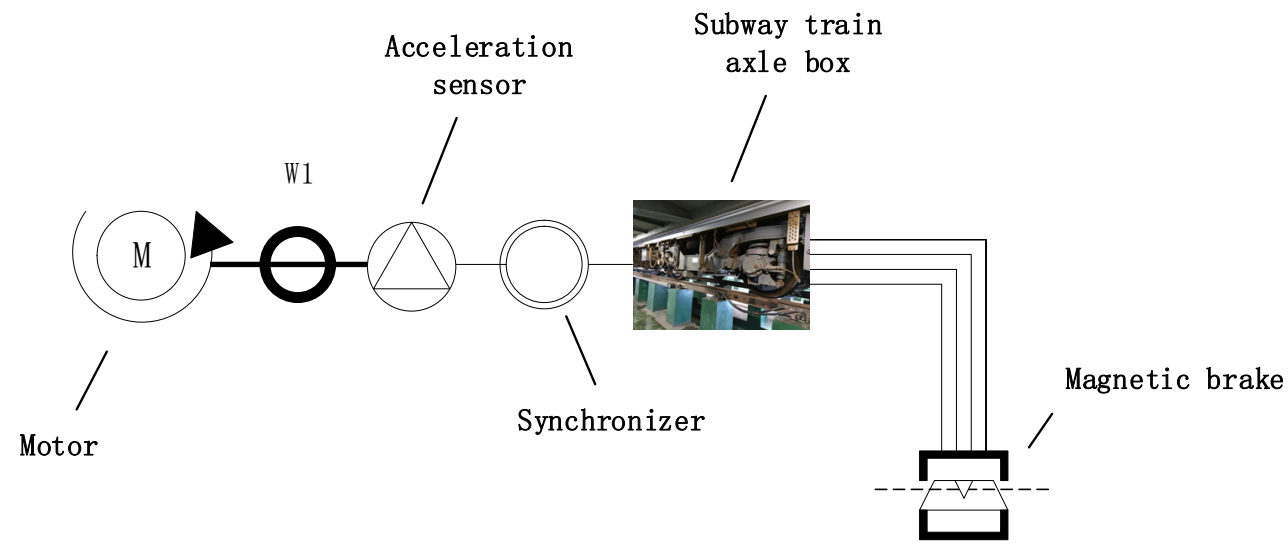

Figure 3. Drive-train dynamic simulator for data collection.

Table 2. Health conditions of bearings in the subway train axle box.

\begin{tabular}{ccccccc}
\hline Category & Health Conditions & $\begin{array}{c}\text { Sampling Points } \\
\text { (per Series) }\end{array}$ & Series & $\begin{array}{c}\text { Total Sampling } \\
\text { Points }\end{array}$ & $\begin{array}{c}\text { Rotation } \\
\text { Speed (rpm) }\end{array}$ & Loads (KN) \\
\hline 1 & Roller fault & 122,581 & 16 & $1,961,296$ & 1752 & $36 / 72$ \\
2 & Inner raceway fault & 125,049 & 16 & $2,000,784$ & 1751 & $36 / 72$ \\
3 & Outer raceway fault & 122,514 & 16 & $1,960,224$ & 1751 & $36 / 72$ \\
4 & Normal & 122,500 & 16 & $1,960,000$ & 1750 & $36 / 72$ \\
5 & Total & - & 64 & $7,882,304$ & - & $36 / 72$ \\
\hline
\end{tabular}

The data processing after the raw signal acquisition is shown in Figure 4. The raw vibration signal collected by the acceleration sensor is sampled to more than 120,000 points per series, The number of sampling points per series for each of the four health conditions is shown in Table 2. By data splitting, the normalized vibration signal is split into several signal fragments, here the size of each segment is set to 800, which can effectively ensure the sparsity of the features and the size of the dataset, and then repeat the operation to complete the splitting of all the data, import the completed splitting data into the GASF for data reconstruction as well as divide the obtained 2D time-series graph into training set and test set, the specific number of samples and testing set samples are shown in Table 3.

Although deep learning is well developed for computer vision, it is difficult to build predictive models when encountering time series. For different raw input data such as 1D vibration signal data, it is necessary to reconstruct the signals of $1 \mathrm{D}$ time series into the form of $2 \mathrm{D}$ images, thus the advantages of computer vision can be fully exploited. The 2D timing diagrams of the four health conditions converted from 1D vibration signals by GASF are shown in Figure 5. 


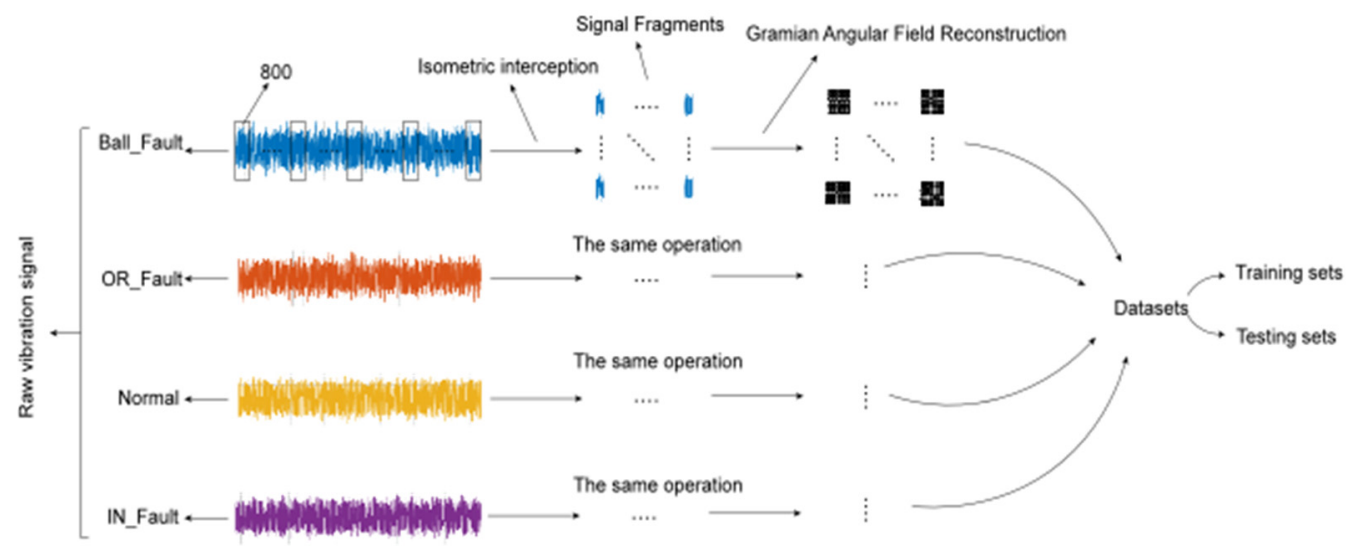

Figure 4. General illustrator of data processing.

Table 3. Dataset size for each kind of health condition.

\begin{tabular}{cccccc}
\hline Fault Category & Roller Fault & Inner Raceway Fault & Outer Raceway Fault & Normal & Total \\
\hline Sample size & 5620 & 5742 & 5505 & 4245 & 21,112 \\
Image size & $128 \times 128$ & $128 \times 128$ & $128 \times 128$ & $128 \times 128$ & $128 \times 128$ \\
$\begin{array}{c}\text { Number of feature } \\
\text { pixel points }\end{array}$ & $92,078,080$ & $94,076,928$ & $90,193,920$ & $69,550,080$ & $345,899,008$ \\
\hline
\end{tabular}

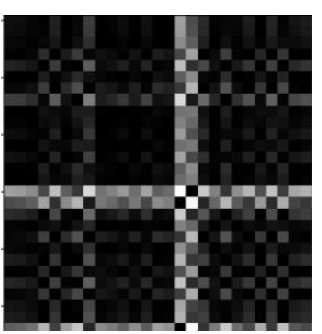

(a)

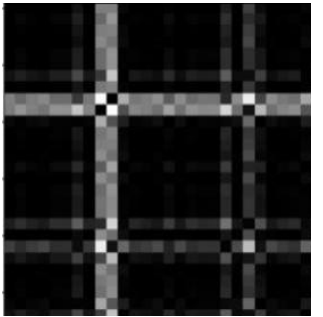

(b)

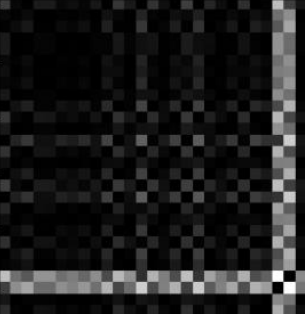

(c)

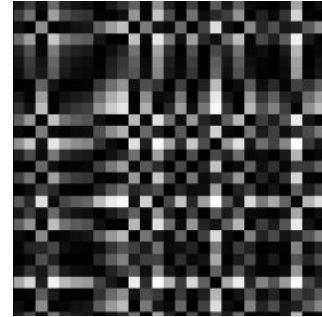

(d)

Figure 5. Two-dimensional fault timing diagram obtained from a 1D vibration signal using GASF. (a) Roller fault, (b) inner raceway fault, (c) outer raceway fault and (d) normal.

\subsection{Hyperparameters Setup}

The four neural networks covered in this paper, including the two residual networks with embedded stacked convolutional kernels, are all implemented based on the tensorflow2.0 framework. Experiments were conducted on a thinkstation with an Intel Xeon W-2223 CPU and an NVIDIA Quadro P220 GPU. In this section, the initialization and setting of hyperparameters are described in detail.

Because there is no clear consensus on the hyperparameter settings for classical CNNs and ResNets [27]. The genetic algorithm (GA) is advanced and outstanding; however, there are hyperparametric results of the optimization of the algorithm itself, which need to be verified experimentally in the next stage, so it is not intuitive. Hence, in this paper, the hyperparameters are set according to the experiment results shown in Figure 6. The experiments are conducted based on the representative VGG-16 and ResNet-152 built in this paper and have shown that when the activation function of the convolution layer is set to ReLU, it converges faster and with higher accuracy than tanh. Moreover, when the loss function is set to mean square error (MSE), the diagnostic accuracy is significantly inferior to that of cross entropy. The effect of the Adam optimizer is similar to RMSprop, so this paper chooses the more commonly used Adam optimizer. In addition, other relevant parameters are shown in Table 4 . The input data shape set to $128 \times 128$ and the depth to three. The meaning of Conv_2 in Table 4 is the number of 2D convolutional layers, activation function_1 refers to the activation function used in the convolutional layer, the ReLU function ensures the sparsity of the network and reduces the interdependence of the 
parameters, alleviating the overfitting. RBU refers to whether identity shortcut mapping is used in the network structure, where 1 means used and 0 means not used.
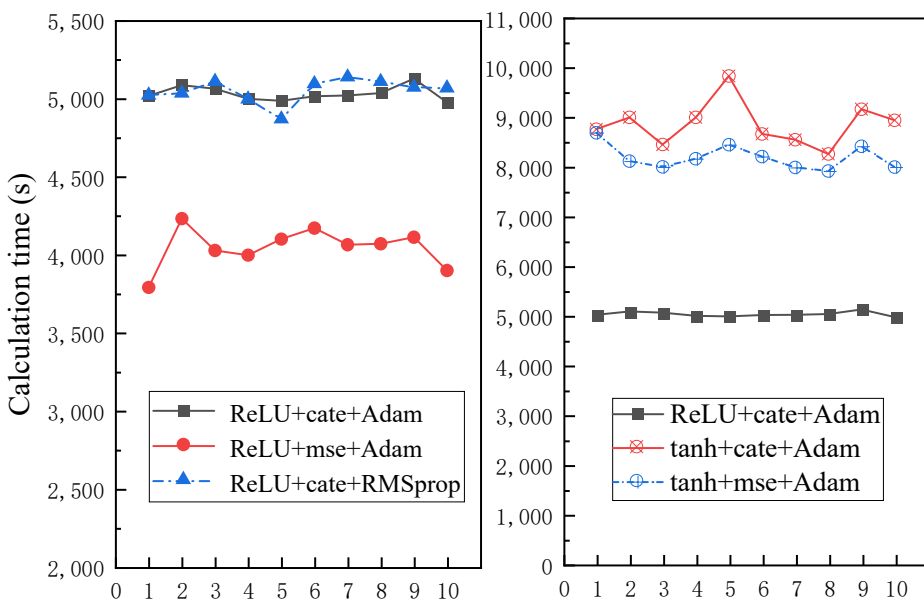

Experiment number (VGG-16)

(a)
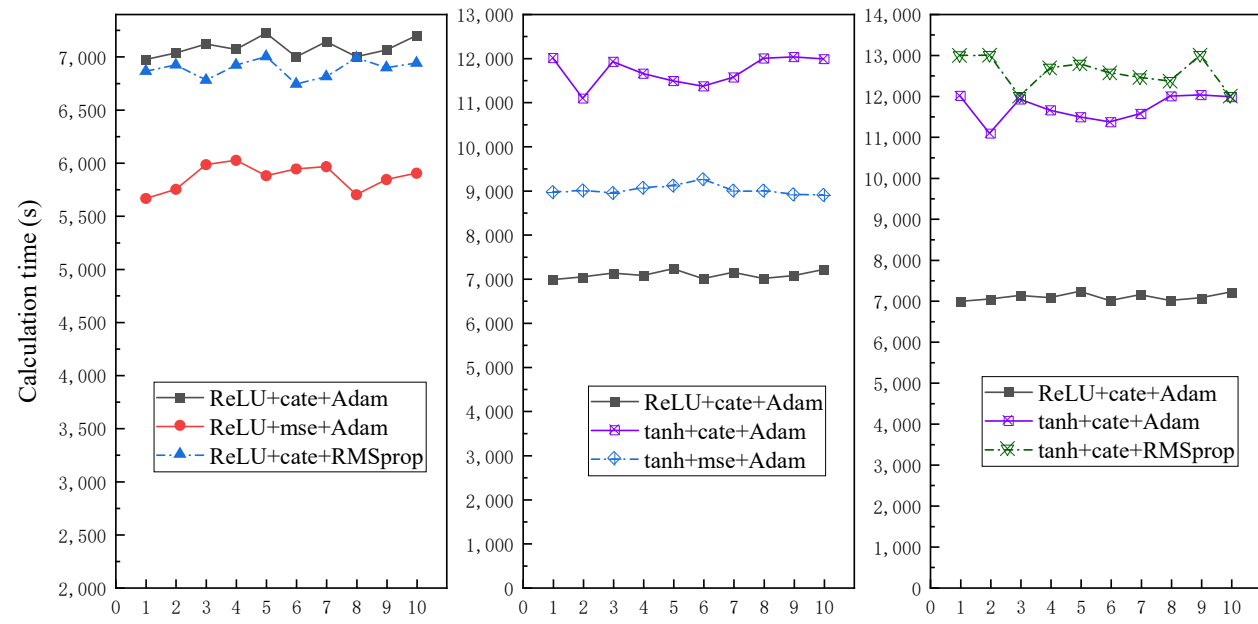

Experiment number (ResNet-152)

(b)

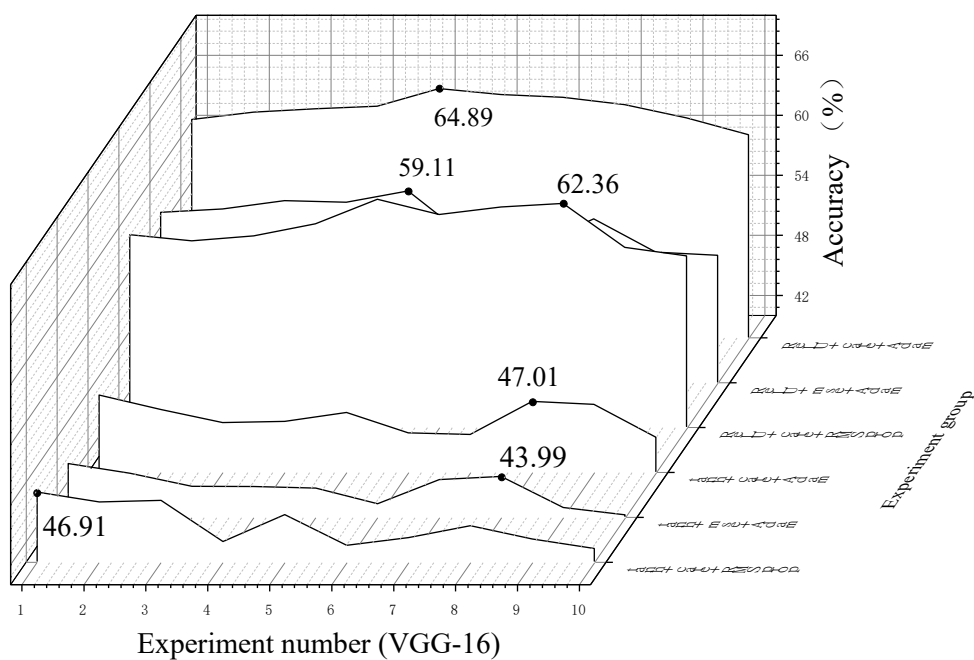

(c)

Figure 6. Cont. 


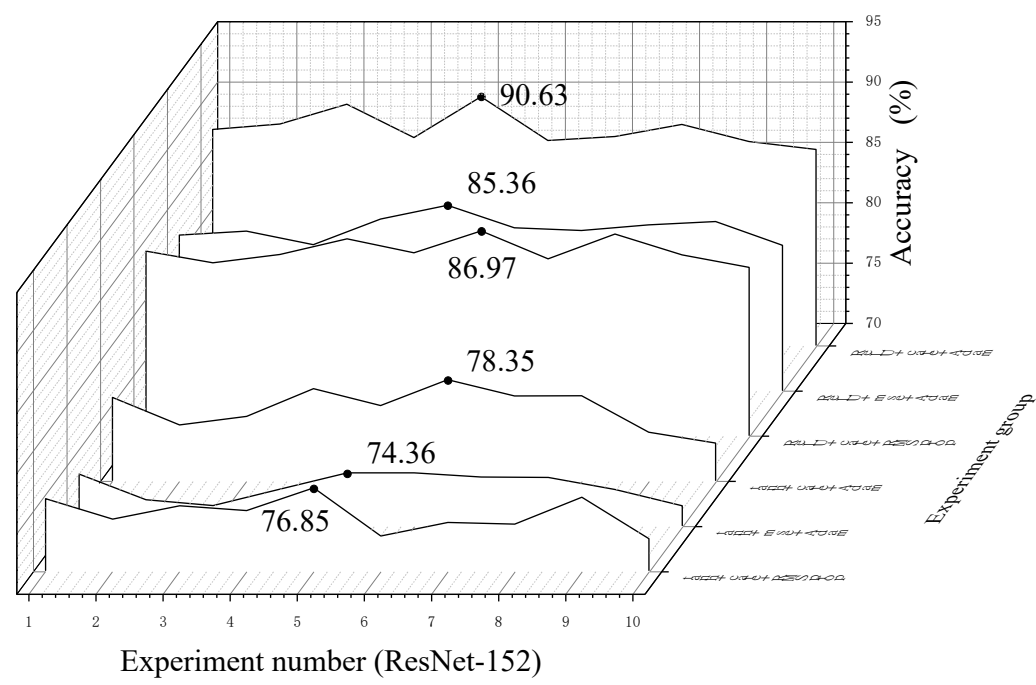

(d)

Figure 6. Effects of several hyperparameter settings in VGG-16 and ResNet-152. (a) Calculation time of VGG-16, (b) calculation time of ResNet-152, (c) accuracy comparison of VGG-16, (d) accuracy comparison of ResNet-152.

Table 4. Architecture-related hyperparameters of the VGG-16, VGG-19, ResNet-50, ResNet-152, ResNet-50-MSRF, and ResNet-152-MSRF in the experiment.

\begin{tabular}{|c|c|c|c|c|c|c|}
\hline Components & VGG-16 & VGG-19 & ResNet-50 & ResNet-152 & ResNet-50-MSRF & ResNet-152-MSRF \\
\hline Input & $128 \times 128 \times 3$ & $128 \times 128 \times 3$ & $128 \times 128 \times 3$ & $128 \times 128 \times 3$ & $128 \times 128 \times 3$ & $128 \times 128 \times 3$ \\
\hline Conv_2 & 13 & 16 & 50 & 152 & 50 & 152 \\
\hline Conv_kērnel & $(3 \times 3,1)$ & $(3 \times 3,1)$ & $(3 \times 3,2)$ & $(3 \times 3,2)$ & $(3 \times 3 \times 3,2)$ & $(3 \times 3 \times 3,2)$ \\
\hline Strides & 1 & 1 & 1 & 1 & 1 & 1 \\
\hline $\mathrm{BN}$ & 15 & 18 & 48 & 150 & 48 & 150 \\
\hline $\begin{array}{l}\text { Activation } \\
\text { function } 1\end{array}$ & ReLU & ReLU & ReLU & ReLU & ReLU & ReLU \\
\hline RBU & 0 & 0 & 1 & 1 & 1 & 1 \\
\hline Activation & softmax & softmax & softmax & softmax & softmax & softmax \\
\hline FC & 3 & 3 & 2 & 2 & 2 & 2 \\
\hline Loss function & Category_crossentropy & Category_crossentropy & Category_crossentropy & Category_crossentropy & Category_crossentropy & Category_crossentropy \\
\hline output & 4 & 4 & 4 & 4 & 4 & 4 \\
\hline Optimizer & Adam & Adam & Adam & Adam & Adam & Adam \\
\hline $\mathrm{Lr}$ & 0.0001 & 0.0001 & 0.0001 & 0.0001 & 0.0001 & 0.0001 \\
\hline Dropout & 0.5 & 0.5 & 0.5 & 0.5 & 0.5 & 0.5 \\
\hline
\end{tabular}

Activation function_2 represents the FC activation function. Softmax function is suitable for multi-classification tasks. The learning rate is also uniformly set to 0.0001 , which is kept as the same as classical ResNets [18]. The use of the FC loss function has been explained in Equation (3) and the number of output nodes in the FC is set to 4, which is the same as the number of health conditions and contains one normal condition and 3 fault states. The batch size has been set to 20 .

\subsection{Comparison of Six Target Diagnostic Models}

As shown in Table 3, the total sample size reached 21,112, in addition to the number of feature pixel points which reached $345,899,008$. Compared with the original data scale, the data scale has increased approximately 42.88 times, so experiments were conducted under a scheme of sixfold cross validation. The total dataset is divided into six subsets, five of them are used as the training set and one as the test set, and then the average accuracy and loss are taken as the experimental results after cross validation. In addition, this experiment is conducted for different classification tasks, and three sets of experiments are conducted to verify the diagnostic performance of the model under different classification tasks. 
The general operation route of the experiment is shown in Figure 7, including the basic principles of GASF and architecture of residual building block (RBB), in which RBB-1 maps the input directly to the output and RBB-2 deals with the input by convolution and BN.

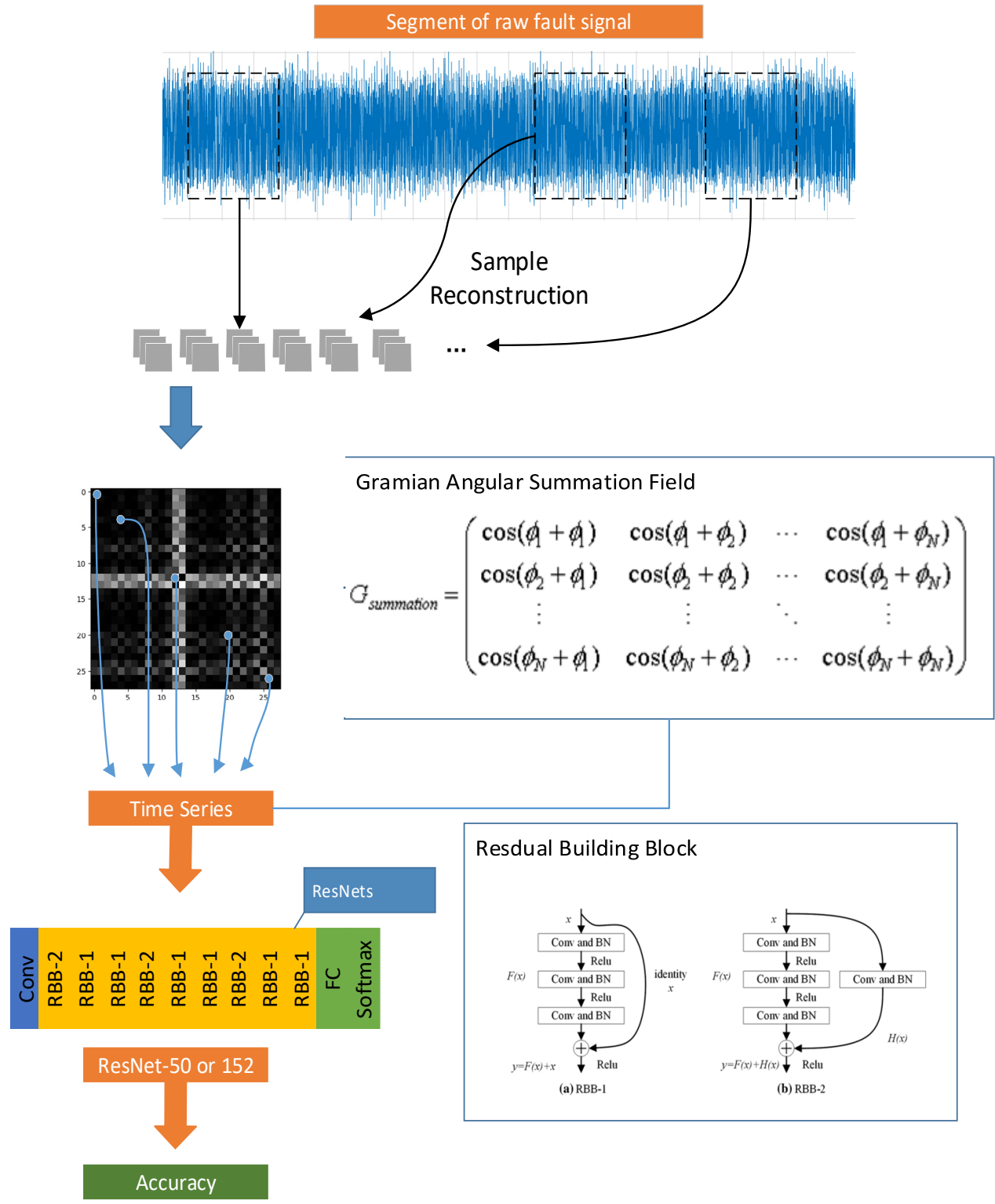

Figure 7. The illustration of research operation route.

Comparison of the average accuracy of the six neural network models trained and validated on four different health condition datasets is shown in Figure 8, and the average accuracies on the validation sets for all cases are given in Table 5. ResNets are significantly superior to the general $\mathrm{CNN}$ in diagnostic performance in terms of network structure alone. Moreover, for large-scale input data with deep features, residual networks show an extraordinary learning ability. The residual network achieves good accuracy in the more difficult quadruple classification task, to over $95 \%$, and is relatively stable in the simpler quadruple classification task, with fluctuations below $0.5 \%$. 


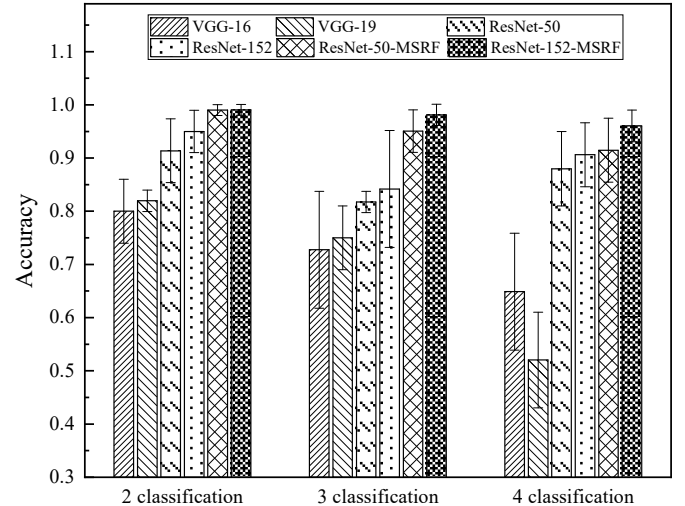

(a)

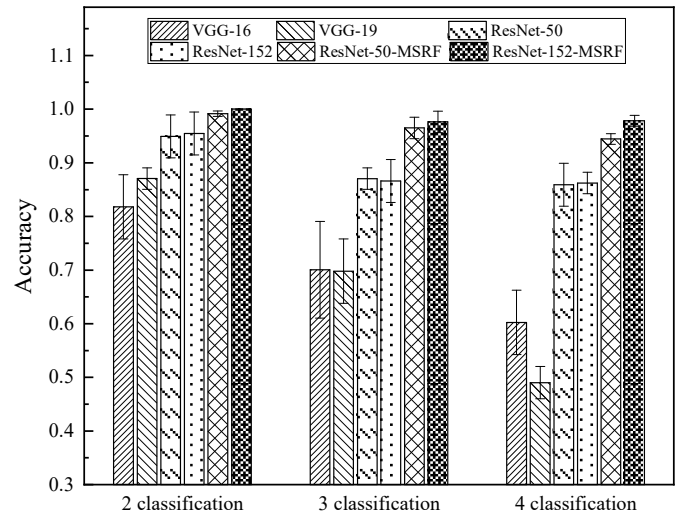

(c)

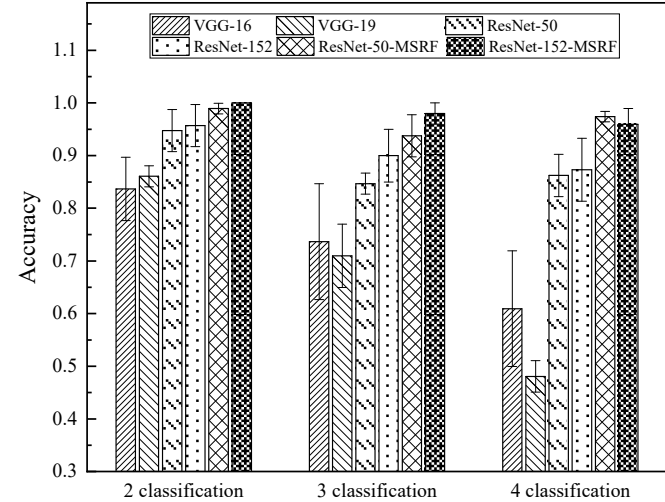

(b)

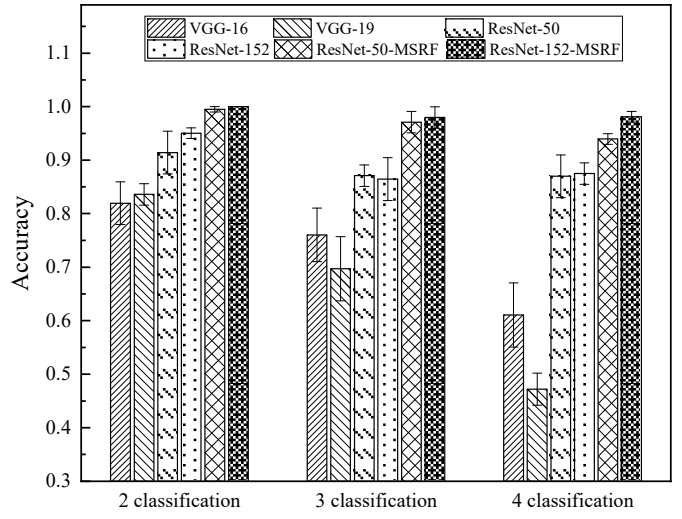

(d)

Figure 8. Comparison of the accuracy of six neural network models trained and validated under four different datasets, (a-d) represent different dataset contents. (a) A total of 3 classifications consists of normal, outer raceway fault and inner raceway fault; 2 classification consists of normal and outer raceway fault, (b) 3 classifications consists of normal, outer raceway fault and roller fault; 2 classifications consists of normal and inner raceway fault, (c) 3 classifications consists of normal, inner raceway fault and roller fault; 2 classifications consists of normal and roller fault, (d) 3 classifications consists of outer raceway fault, inner raceway fault and roller fault; 2 classifications consists of inner raceway fault and roller fault.

Table 5. Average accuracy of the results in Figure 8 (including the lowest and highest accuracy).

\begin{tabular}{cccc}
\hline Method & $\begin{array}{c}\text { 2 Classification } \\
\text { Accuracy (\%) }\end{array}$ & $\begin{array}{c}\text { 3 Classification } \\
\text { Accuracy (\%) }\end{array}$ & $\begin{array}{c}\text { 4 Classification } \\
\text { Accuracy (\%) }\end{array}$ \\
\hline VGG-16 & $81.85_{-1.85}^{+1.82}$ & $73.13_{-3.06}^{+2.91}$ & $61.79_{-1.53}^{+3.10}$ \\
VGG-19 & $84.68_{-2.09}^{+2.38}$ & $71.37_{-1.66}^{+3.64}$ & $49.09_{-1.88}^{+2.95}$ \\
ResNet-50 & $93.18_{-1.81}^{+1.75}$ & $85.14_{-3.40}^{+1.96}$ & $86.78_{-0.87}^{+1.22}$ \\
ResNet-152 & $95.30_{-0.30}^{+0.39}$ & $86.81_{-2.63}^{+3.19}$ & $87.92_{-1.68}^{+2.71}$ \\
ResNet-50-MSRF & $99.15_{-0.22}^{+0.34}$ & $95.61_{-1.86}^{+1.50}$ & $94.32_{-2.85}^{+3.09}$ \\
ResNet-152-MSRF & $99.77_{-0.70}^{+0.23}$ & $97.93_{-0.31}^{+0.20}$ & $96.99_{-1.04}^{+1.13}$ \\
\hline
\end{tabular}

The average calculation time of six different neural network models is shown in Table 6, where the validation batch is set to 10, and that on the training set is set to 20, as a result, ResNet-152-MSRF takes more time than others. Obviously, comparing the number of trainable participants of the network in Table 1, it is clear that it is related to the size of 
the number of parameters. It is worth noting that both ResNet-50 and ResNet-152 with stacked convolutional kernels embedded (i.e., ResNet-50-MSRF and ResNet-152-MSRF) saw their computation times rise by only $8.201 \%$ and $5.577 \%$, respectively.

Table 6. Average calculation time of six different neural network models.

\begin{tabular}{ccccc}
\hline Method & $\begin{array}{c}\text { Training/Testing } \\
\text { Batch Size }\end{array}$ & Epochs & $\begin{array}{c}\text { Total Calculation } \\
\text { Time (s) }\end{array}$ & $\begin{array}{c}\text { Calculation Time } \\
\text { per Step (s) }\end{array}$ \\
\hline VGG-16 & $20 / 10$ & 100 & 5019 & 50.19 \\
VGG-19 & $20 / 10$ & 100 & 6467 & 64.67 \\
ResNet-50 & $20 / 10$ & 100 & 2536 & 25.36 \\
ResNet-152 & $20 / 10$ & 100 & 6974 & 69.74 \\
ResNet-50-MSRF & $20 / 10$ & 100 & 2744 & 27.44 \\
ResNet-152-MSRF & $20 / 10$ & 100 & 7363 & 73.63 \\
\hline
\end{tabular}

\subsection{Comparison between ResNet-152-MSRF and ResNet-50-MSRF}

As shown in Table 5, the average diagnostic accuracy of ResNet-152-MSRF is $2.67 \%$ higher than that of ResNet-50-MSRF in the four-classification task, $2.28 \%$ higher in the three-classification task, and $0.62 \%$ higher in the two-classification task.

$\mathrm{K}$. He et al. verified that neural networks lead to lower accuracy when the depth is significantly increased [18]. However, as shown in Figure 9, ResNet-152-MSRF is more stable than the ResNet-50-MSRF, with no significant fluctuations in diagnostic accuracy or error. In contrast with Figure 9a,c. ResNet-50-MSRF achieves faster accuracy and error convergence than ResNet-152-MSRF on the training set, reaching more than $80 \%$ training accuracy after 15 iterations, and then combined with the calculation time comparison in Table 6.

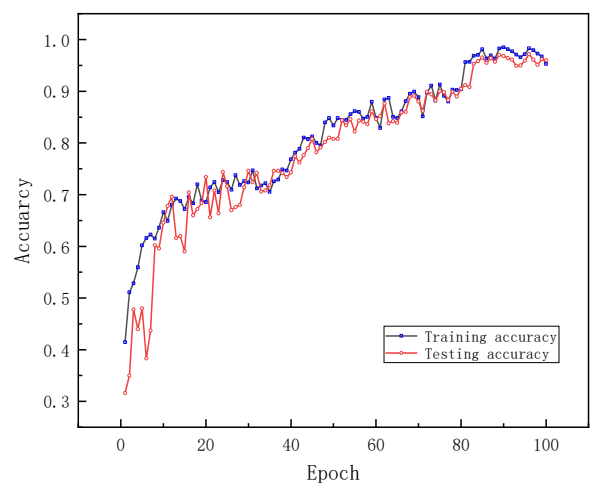

(a)

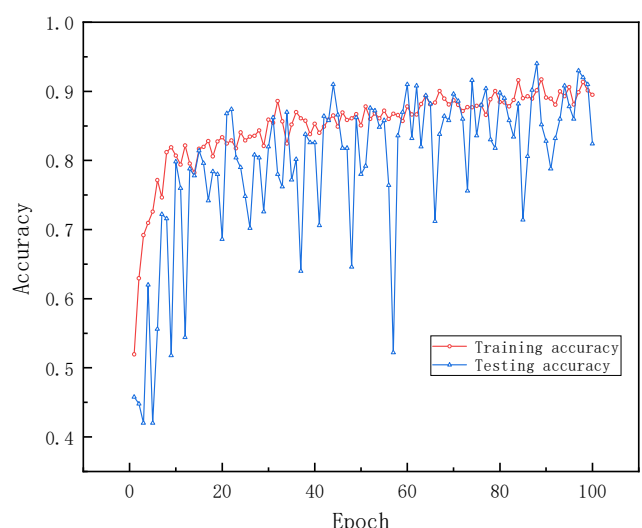

(c)

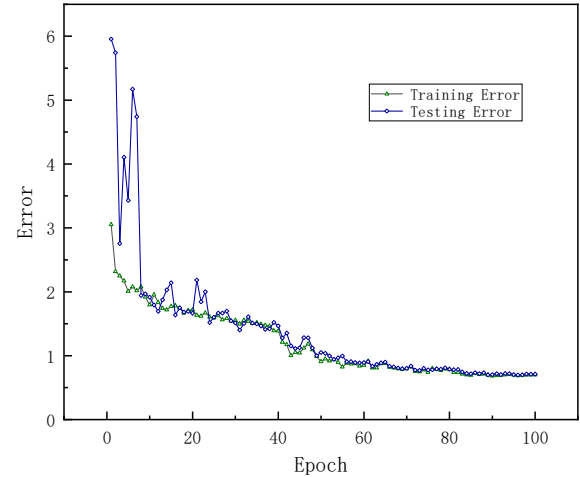

(b)

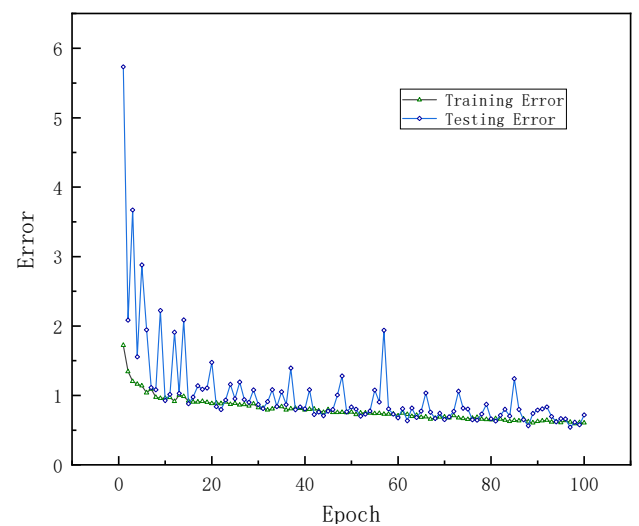

(d)

Figure 9. Accuracy and error comparison between ResNet-50-MSRF and ResNet-152-MSRF. (a) ResNet-152-MSRF training and testing accuracy, (b) ResNet-152-MSRF training and testing error, (c) ResNet-50-MSRF training and testing accuracy, (d) ResNet-50-MSRF training and testing error. 


\section{Conclusions}

Rapid diagnosis of bearings inside axle boxes of subway trains is an important task to accelerate the modular maintenance of the whole train. This article mainly develops a fault diagnosis method based on ResNet-152-MSRF. In addition, the traditional 1D fault signal is reconstructed using GASF, so that 2D convolution layers can be built to facilitate feature extraction.

(1) Evidenced by the experiments, ResNet-50 and ResNet-152 improved by 24.99\%, $37.69 \%, 26.13 \%$ and $38.83 \%$ relative to VGG-16 and VGG-19, respectively. Additionally, the result indicates that networks with RBB are more suitable for large-scale deep feature extraction;

(2) Evidenced by the data reconstruction, the scale of the obtained data is increased by about 42.88 times compared to the previous 1D time series signal, which is effective for data enhancement;

(3) By embedding a multi-layered receptive field, the developed ReNet-152-MSRF enhances the accuracy by $9.07 \%$ compared to ResNet-152, and time cost increases nonsignificantly. ResNet-152-MSRF has a $2.67 \%$ and $1.87 \%$ higher average diagnostic accuracy, respectively, than ResNet-50-MSRF in different tasks. This suggests that deeper networks do not necessarily affect accuracy and perform well when trained on reconstructed fault data.

In the future, the method proposed in this paper will be verified in more real-time monitoring platform and more accurate models will be obtained.

Author Contributions: Proposing the original technical line of thought, H.Y.; methodology, H.Y. and X.M; Software, H.Y. and X.M.; formal analysis, H.Y.; investigation, H.Y.; resources, H.Y.; data curation, H.Y.; writing—original draft preparation, H.Y.; writing—review and editing, H.Y., X.M. and H.W.; visualization, H.Y.; supervision, X.M. and H.W.; project administration, X.M.; funding acquisition, X.M. All authors have read and agreed to the published version of the manuscript.

Funding: This research was funded by [NSFC] 52175465.

Acknowledgments: All individuals included in this section have consented to the acknowledgement.

Conflicts of Interest: The authors declare no conflict of interest. Miao gave reference to the technical route of this work, participated in the review and editing of the paper, and provided guidance on the experimental platform and data analysis, with no conflict of interest. Wang provided guidance on the technical direction of this work, with no conflict of interest.

\section{References}

1. Liu, G.F.; Hou, Z.S. Adaptive Iterative Learning Control for Subway Trains Using Multiple-Point-Mass Dynamic Model under Speed Constraint. IEEE Trans. Intell. Transp. Syst. 2021, 22, 1388-1400. [CrossRef]

2. Wang, Y.; Yang, Z.; Pang, J. Statistical analysis of urban rail transit lines in 2017 China-Express delivery of annual report on urban rail transit. Urban Mass Transit 2018, 21, 1-6.

3. Sun, J.W.; Zhang, A.; Zhou, Z.X. Research on Urban Rail Transit Promoting the Process of Urbanization. China Soft Sci. 2020, 6, 96-111.

4. Zhang, D.; Entezami, M.; Stewart, E.; Roberts, C.; Yu, D. Adaptive fault feature extraction from wayside acoustic signals from train bearings. J. Sound Vib. 2018, 425, 221-238. [CrossRef]

5. Arturo, G.; Roberto, P.; Paul, B. Sustainable urban rail systems: Strategies and technologies for optimal management of regenerative braking energy. Energy Convers. Manag. 2013, 75, 1879-2227.

6. Cao, H.; Fan, F.; Zhou, K.; He, Z. Wheel-bearing fault diagnosis of trains using empirical wavelet transform. Measurement 2016, 82, 439-449. [CrossRef]

7. Zhang, Z.; Entezami, M.; Stewart, E.; Roberts, C. Enhanced fault diagnosis of roller bearing elements using a combination of empirical mode decomposition and minimum entropy deconvolution. J. Mech. Eng. Sci. 2017, 231, 655-671. [CrossRef]

8. Lei, Y.; Lin, J.; Zuo, M.J.; He, Z. Condition monitoring and fault diagnosis of planetary gearboxes: A review. Measurement 2014, 48, 292-305. [CrossRef]

9. Cui, M.; Wang, Y.; Lin, X.; Zhong, M. Fault Diagnosis of Rolling Bearings Based on an Improved Stack Autoencoder and Support Vector Machine. IEEE Sens. J. 2021, 21, 4927-4937. [CrossRef]

10. Rai, A.; Upadhyay, S.H. A review on signal processing techniques utilized in the fault diagnosis of rolling element bearings. Tribol. Int. 2016, 96, 289-306. [CrossRef] 
11. Liu, R.; Yang, B.; Zio, E.; Chen, X. Artificial intelligence for fault diagnosis of rotating machinery: A review. Mech. Syst. Signal Processing 2018, 108, 33-47. [CrossRef]

12. Roy, S.; Menapace, W.; Oei, S.; Luijten, B.; Fini, E.; Saltori, C.; Huijben, I.; Chennakeshava, N.; Mento, F.; Sentelli, A.; et al. Deep Learning for Classification and Localization of COVID-19 Markers in Point-of-Care Lung Ultrasound. IEEE Trans. Med. Imaging 2020, 39, 2676-2687. [CrossRef]

13. Baltruschat, I.M.; Nickisch, H.; Grass, M.; Knopp, T.; Saalbach, A. Comparison of Deep Learning Approaches for Multi-Label Chest X-Ray Classification. Sci. Rep. 2019, 9, 6381. [CrossRef]

14. Lee, K.B.; Shin, H.S. An Application of a Deep Learning Algorithm for Automatic Detection of Unexpected Accidents Under Bad CCTV Monitoring Conditions in Tunnels. In Proceedings of the 2019 International Conference on Deep Learning and Machine Learning in Emerging Application (Deep-ML), Istanbul, Turkey, 26-28 August 2019; pp. 7-11.

15. Dikbayir, H.S.; Ïbrahim Bülbül, H. Deep Learning Based Vehicle Detection from Aerial Images. In Proceedings of the 2020 19th IEEE International Conference on Machine Learning and Applications (ICMLA), Miami, FL, USA, 14-17 December 2020; pp. 956-960.

16. Shao, H.D.; Jiang, H.K.; Zhang, X.; Niu, M.G. Rolling bearing fault diagnosis using an optimization deep belief network. Meas. Sci. Technol. 2015, 26, 115002. [CrossRef]

17. Goodfellow, I.; Benigo, Y.; Courville, A. Deep Learning; MIT Press: Cambridge, MA, USA, 2016.

18. He, K.M.; Zhang, X.; Ren, S.; Sun, J. Deep Residual Learning for image recognition. In Proceedings of the IEEE Conference on Computer Vision and Pattern Recognition (CVPR), Seattle, WA, USA, 27-30 June 2016; pp. 770-778.

19. Gao, S.H.; Cheng, M.M.; Zhao, K.; Zhang, X.Y.; Yang, M.H.; Torr, P. Res2Net: A New Multi-Scale Backbone Architecture. IEEE Trans. Pattern Anal. Mach. Intell. 2021, 43, 652-662. [CrossRef]

20. Paoletti, M.E.; Haut, J.M.; Fernandez-Beltran, R.; Plaza, J.; Plaza, A.J.; Pla, F. Deep Pyramidal Residual Networks for SpectralSpatial Hyperspectral Image Classification. IEEE Trans. Geosci. Remote Sens. 2019, 57, 740-754. [CrossRef]

21. Li, B.; He, Y. An Improved ResNet Based on the Adjustable Shortcut Connections. IEEE Access 2018, 6, 18967-18974. [CrossRef]

22. Wen, L.; Li, X.; Gao, L. A transfer convolutional neural network for fault diagnosis based on ResNet-50. Neural Comput. Appl. 2020, 32, 6111-6124. [CrossRef]

23. Rasp, S.; Thuerey, N. Data-Driven Medium-Range Weather Prediction with a Resnet Pretrained on Climate Simulations: A New Model for WeatherBench. J. Adv. Modeling Earth Syst. 2021, 13, 1942-2466. [CrossRef]

24. Zhou, C.; Song, J.; Zhou, S.; Zhang, Z.; Xing, J. COVID-19 Detection Based on Image Regrouping and ResNet-SVM Using Chest X-Ray Images. IEEE Access 2021, 9, 81902-81912. [CrossRef]

25. Zhao, M.; Kang, M.; Tang, B.; Pecht, M. Deep Residual Networks with Dynamically Weighted Wavelet Coefficients for Fault Diagnosis of Planetary Gearboxes. IEEE Trans. Ind. Electron. 2018, 65, 4290-4300. [CrossRef]

26. Zhao, M.; Zhong, S.; Fu, X.; Tang, B.; Dong, S.; Pecht, M. Deep Residual Networks with Adaptively Parametric Rectifier Linear Units for Fault Diagnosis. IEEE Trans. Ind. Electron. 2021, 68, 2587-2597. [CrossRef]

27. Zhao, M.; Zhong, S.; Fu, X.; Tang, B.; Pecht, M. Deep Residual Shrinkage Networks for Fault Diagnosis. IEEE Trans. Ind. Inform. 2020, 16, 4681-4690. [CrossRef]

28. Wu, S.T.; Zhong, S.H.; Liu, Y. Deep residual learning for image steganalysis. Multimed. Tools Appl. 2018, 77, 10437-10453. [CrossRef]

29. Loffe, S.; Szegedy, C. Batch normalization: Accelerating deep network training by reducing internal covariate shift. In Proceedings of the 32nd International Conference on Machine Learning, Lille, France, 6-11 July 2015; pp. 448-456.

30. Wu, Y.X.; He, K.M. Group Normalization. Int. J. Comput. Vis. 2020, 128, 742-755. [CrossRef] 